Pacific Journal of Mathematics

ON THE COUNTABLE UNION OF CELLULAR 


\title{
ON THE COUNTABLE UNION OF CELLULAR DECOMPOSITIONS OF $n$-MANIFOLDS
}

\author{
W. VOXMAN
}

\begin{abstract}
Suppose that $G_{1}, G_{2} \ldots$ are cellular upper semicontinuous decompositions of an $n$-manifold with boundary $M(n \neq 4)$ such that for $i=1,2, \cdots, M / G_{i}$ is homeomorphic to $M$. Let $G$ be the decomposition of $M$ obtained from the decomposition of $G_{i}$ in the following manner. A set $g$ belongs to $G$ if and only if $g$ is a nondegenerate element of some $G_{i}$ or $g$ is a point in $M-\left(\bigcup_{i=1}^{\infty} H_{G_{i}}^{*}\right)$. It will be shown that if the various decompositions fit together in a "continuous" manner and if $G$ is an upper semicontinuous decomposition of $M$, then $M / G$ is homeomorphic to $M$.
\end{abstract}

Our principal result thus extends previous results obtained by the author ([6], [7]) and Lamoreaux [4], by removing the 0-dimensionality restriction in [6] or, alternatively, by eliminating the finiteness condition in [7]. Furthermore, with the aid of recent work of Siebenmann [5], generalizations to $n$-manifolds $(n \neq 4)$ may be made. As was observed in [7], some conditions must be imposed on the manner in which the decompositions are pieced together. The example described by Bing in [2] demonstrates that the continuity condition to be described below is a necessary one.

Notation and terminology. Suppose $G$ is an upper semicontinuous decomposition of a topological space, $X$. Then $X / G$ will denote the associated decomposition space, $P$ will denote the natural projection map from $X$ onto $X / G$, and $H_{G}$ will denote the collection of nondegenerate elements of $G$. If $U$ is an open subset of $X$, then $U$ is said to be saturated (with respect to $G$ ) in case $U=P^{-1}[P[U]]$. If $\mathscr{U}$ is a covering of a subset of $X$, then $P[\mathscr{U}]=\{P[U]: U \in \mathscr{U}\}$.

The statement that $M$ is an $n$-manifold with boundary means that $M$ is a separable metric space such that each point of $M$ has a neighborhood which is an $n$-cell. If $A$ is a subset of $M$, then $A$ is cellular in $M$ if there exists a sequence $C_{1}, C_{2}, \cdots$ of $n$-cells in $M$ such that (1) for each positive integer $i, C_{i+1} \subset$ Interior $C_{i}$, and (2) $\bigcap_{i=1}^{\infty} C_{i}=A$. If $M$ is an $n$-manifold with boundary, the statement that $G$ is cellular decomposition of $M$ means that $G$ is an upper semicontinuous decomposition of $M$ and each nondegenerate element of $G$ is a cellular subset of $M$.

If $M$ is a metric space, $A$ a subset of $M$, then $S_{\varepsilon}(A)$ denotes the $\varepsilon$-neighborhood of $A$ and $\mathrm{Cl} A$ denotes the closure of $A$ in $M$. If $K$ 
is a collection of subsets of $M$, then $K^{*}=\bigcup\{k: k \in K\}$. The word map will always be used to indicate a continuous function. If $\mathscr{C}$ is a collection of subsets of $M$ and $A \subset M$, then

$$
\operatorname{St}(A, \mathscr{C})=\bigcup\{U \in \mathscr{W}: A \cap U \neq \varnothing\} \text {. }
$$

The main result. The principal theorem will be proved by means of repeated applications of the Lemma which appears below. We say that a cellular decomposition $G$ of a manifold $M$ satisfies condition $S$ if for each saturated open cover $\mathscr{C}$ of $H_{G}^{*}$, there exists a closed map $h$ from $M$ onto $M$ such that (1) $G=\left\{h^{-1}(x): x \in M\right\}$, (2) if $x \in M-\mathscr{Q}^{*}$, then $h(x)=x$, and (3) for each $g \in G$ and $g \subset \mathscr{Q}^{*}$, there exists a $U \in \mathscr{W}$ such that $g \cup h(g) \subset U$.

Lemma 1. Suppose $G$ is a cellular decomposition of an $n$-manifold with boundary $M(n \neq 4)$. Then $M / G$ is homeomorphic to $M$ if and only if $G$ satisfies condition $S$.

Proof. Clearly if $G$ satisfies condition $S$, then $M / G$ is homeomorphic to $M$. Suppose now that $M / G$ is homeomorphic to $M$ and that $\mathscr{Q}_{6}$ is a saturated open cover of $H_{G}^{*}$. Without loss of generality we may assume that $\mathscr{\mathscr { C }}$ is locally finite. Suppose $x \in \mathscr{Q}^{*}$ and $U_{1}, \cdots, U_{n}$ are those sets in $\mathscr{C}$ which contain $x$. Set

$$
\varepsilon_{x}=\max \left\{d\left(P(x), M / G-P\left[U_{1}\right]\right), \cdots, d\left(P(x), M / G-P\left[U_{n}\right]\right)\right\}
$$

and define $f_{1}(x)=\varepsilon_{x} / 2$. Then $f_{1}$ is a lower semicontinuous function from $\mathscr{Q}^{*}$ into $(0, \infty)$, and, hence, there exists a continuous map $f_{2}$ from $\mathscr{Q}^{*}$ into $(0, \infty)$ such that $0<f_{2}<f_{1}$. For $x \in \mathscr{U}^{*}$, define $f_{3}(x)$ to be $d\left(P(x), M / G-P\left[\mathscr{C}^{*}\right]\right)$, and finally define $f(x)$ to be min $\left\{f_{2}(x), f_{3}(x)\right\}$. Siebenman's projection approximation theorem [5] may be applied to find a homeomorphism $k$ from $\mathscr{C}^{*}$ onto $P\left[\mathscr{C}^{*}\right]$ such that $d(P(x), k(x))<f(x)$ for each $x \in \mathscr{U}^{*}$. Then $h=k^{-1} P$ is the desired map. To see this we need only check that for $g \in G$ and $g \subset \mathscr{U}^{*}$, there is a $U \in \mathscr{Q}$ such that $h(g) \cup g \subset U$. Let $y=k^{-1} P(g)$. By our construction there exists a $U \in \mathscr{U}$ such that both $P(y)$ and $k(y)$ belong to $P[U]$. But $k(y)=P(g)$; therefore, $y$ and $g$ belong to $U$, which completes the proof.

Suppose $M$ is a metric space and $K$ is a collection of mutually disjoint subsets of $M$. If $g \in K$, then $K$ is said to be continuous at $g$ in case for each positive number $\varepsilon$, there exists an open subset $V$ of $M$ containing $g$ such that if $g^{\prime} \in K$ and $g^{\prime} \cap V \neq \varnothing$, then $g \subset S_{\varepsilon}\left(g^{\prime}\right)$ and $g^{\prime} \subset S_{\varepsilon}(g)$.

THEOREM 1. Suppose $G_{1}, G_{2}, \cdots$ are cellular decompositions of an n-manifold with boundary $M(n \neq 4)$ such that 
(1) If $g \in H_{G_{i}}$ and $g \cap H_{G_{j}}^{*} \neq \varnothing$, then $g \in H_{G_{j}}$.

(2) For each $k=1,2, \cdots$, if $g \in H_{G_{k}}$, then $\left\{H_{G_{i}}: i \neq k\right\} \cup\{g\}$ is continuous at $g$.

(3) For $i=1,2, \cdots, M / G_{i}$ is homeomorphic to $M$.

(4) $G=\left\{g: g \in \bigcup_{i=1}^{\infty} H_{G_{i}}\right.$ or $g$ is a point of $\left.M-\left(\bigcup_{i=1}^{\infty} H_{G_{i}}^{*}\right)\right\}$ is an upper semicontinuous decomposition of $M$.

Then $M / G$ is homeomorphic to $M$.

Proof. We show that $G$ satisfies condition $S$. Let $\mathscr{W}$ be a saturated open cover of $H_{G}^{*}$. The required function $h$ will be defined as a limit of a sequence of closed, onto maps which are obtained in the following steps.

Step 1. Let $K_{1}=\{p \in M$ : there exists a sequence of nondegenerate elements, each from a different $H_{G_{i}}$, which converges to $p$. Note that $K_{1}$ is a closed subset of $M$. We construct a saturated (with respect to $G$ ) open refinement of $\mathscr{W}$ which covers $H_{G}^{*}$ and misses $K_{1}$. For each $g \in H_{G}$, let $U_{g}$ be saturated open set with compact closure such that

(1) If $\varepsilon_{g}=\min \left\{\operatorname{diam} g, 1 / 2 d\left(g, K_{1}\right), 1\right\}$, then $U_{g} \subset S_{\varepsilon_{g}}(g)$.

(2) If $g_{i} \in H_{G_{i}}$ and $g_{j} \in H_{G_{j}}(i \neq j)$ and $g_{i}$ and $g_{j}$ are contained in $U_{g}$, then $1 / 2 \operatorname{diam} g_{i}<\operatorname{diam} g_{j}<3 / 2 \operatorname{diam} g_{i}$.

(3) $U_{g}$ is contained in some $W \in \mathscr{W}$ which contains $g$.

Parts (1) and (2) are possible because of the continuity condition imposed on the decompositions. Define $\mathscr{U}_{1}^{\prime}=\left\{U_{g}: g \in H_{G}\right\}$. Let $\mathscr{U}_{1}$ be a saturated open locally finite star refinement of $\mathscr{Q}_{1}^{\prime}$ and $\mathscr{V}_{1}=$ $\left\{U \in \mathscr{U}_{1}: U \cap H_{G_{1}}^{*} \neq \varnothing\right\}$. Observe that it follows from (1) that if $p \in K_{1}$, then $p \notin \mathscr{U}_{1}^{*}$. Furthermore, from (1) and (2) we have that if $p \in K_{1}$ and $\left\{x_{i}\right\}$ is a sequence of points in $\mathscr{L}_{1}^{*}$ which converge to $p$, then the sequence $\left\{\mathrm{St}\left(x_{i}, \mathscr{U}_{1}\right)\right\}$ also converges to $p$.

By Lemma 1, there exists a closed map $h_{1}$ from $M$ onto $M$ such that

(1) $G_{1}=\left\{h_{1}^{-1}(x): x \in M\right\}$.

(2) If $x \in M-\mathscr{V}_{1}^{*}$, then $h_{1}(x)=x$.

(3) If $g \in G_{1}$ and $g \subset \mathscr{Q}_{1}^{*}$, then there exists a set of $U \in \mathscr{L}_{1}$ such that $g \cup h_{1}(g) \subset U$.

In addition, since $\mathscr{U}_{1}$ is saturated with to respect to $G$, part (3) holds for all $g \in G$ which are contained in $\mathscr{U}_{1}^{*}$.

Step 2. The decomposition $G_{2}^{\prime}=\left\{h_{1}(g): g \in G_{2}\right\}$ is clearly cellular and upper semicontinuous. Let $P^{\prime}$ be the projection map from $M$ onto $M / G_{2}^{\prime}$ and $P$ the projection map from $M$ onto $M /\left(G_{1} \cup G_{2}\right)$. Then $P^{\prime} h_{1} P^{-1}$ is readily seen to be a homeomorphism from $M /\left(G_{1} \cup G_{2}\right)$ onto 
$M / G_{2}^{\prime}$. But it was shown in [7] that $M /\left(G_{1} \cup G_{2}\right)$ is homeomorphic to $M$ (using Siebenman's generalization [5] of Armentrout's "projection approximation" theorem [1], the results of [7] may be extended to $n$-manifolds for $n \neq 4)$.

Let $K_{2}=\{p \in M$ : there exists a sequence of nondegenerate elements, each from a different $H_{h_{1}\left[G_{i}\right]}$, which converges to $p$. We construct a saturated (with respect to $h_{1}[G]$ ) open refinement of $h_{1}\left[\mathscr{C}_{1}\right]$ which covers $H_{h_{1}[G]}$ and misses $K_{2}$. Suppose $g^{\prime}=h_{1}(g)$ where $g \in H_{G}$ $H_{G_{1}}$. Choose $U_{g^{\prime}}$ to be saturated (with respect to $h_{1}[G]$ ) open set such that

(1) If $\varepsilon_{g^{\prime}}=\min \left\{\operatorname{diam} g^{\prime}, 1 / 2 d\left(g^{\prime}, K_{2}\right), 1 / 2\right\}$, then $U_{g}, \subset S_{\varepsilon_{g^{\prime}}}\left(g^{\prime}\right)$.

(2) If $g_{i} \in H_{h_{1}\left[G_{i}\right]}$ and $g_{j} \in H_{h_{1}\left[G_{j}\right]}(i \neq j)$ and $g_{i}$ and $g_{j}$ are contained in $U_{g^{\prime}}$, then $1 / 2 \operatorname{diam} g_{i}<\operatorname{diam} g_{j}<3 / 2 \operatorname{diam} g_{i}$.

(3) $h_{1}^{-1}\left(U_{g^{\prime}}\right) \subset S_{1 / 4}(g)$.

(4) If $W=\bigcap\left\{U: U \in h_{1}\left[\mathscr{U}_{1}\right]\right.$ and $\left.h_{1}(g) \subset U\right\}$, then $U_{g^{\prime}} \subset W$.

(5) If $V \in \mathscr{Q}_{1}$ and $g \cup h_{1}(g) \subset V$, then $U_{g}, \subset V$.

(6) $U_{g^{\prime}} \cap \mathrm{Cl}\left(h_{1}\left[H_{G_{1}}^{*}\right]\right)=\varnothing$.

Let $\mathscr{U}_{2}^{\prime}=\left\{U_{g^{\prime}}: g^{\prime} \in H_{h_{1}[G]}\right\}$ and let $\mathscr{U}_{2}$ be a saturated open locally finite star refinement of $\mathscr{\mathscr { L }}_{2}^{\prime}$ covering $H_{h_{1}[G]}^{*}$. Let

$$
\mathscr{\mathscr { V }}_{2}=\left\{U \in \mathscr{\mathscr { C }}_{2}: U \cap H_{h_{1}\left[G_{2}\right]}^{*} \neq \varnothing\right\} \text {. }
$$

Note that $h_{1}^{-1}\left(\mathscr{C}_{1}^{*}\right) \subset S_{1 / 2}\left(H_{G}^{*}\right)$ and $h_{1}^{-1}\left(\mathscr{V}_{2}^{*}\right) \subset S_{1 / 2}\left(H_{G_{2}}^{*}\right)$.

By Lemma 1 , there is a closed map $h_{2}$ from $M$ onto $M$ such that

(1) $G_{2}^{\prime}=\left\{h_{2}^{-1}(x): x \in M\right\}$.

(2) If $x \in M-\mathscr{q}_{2}^{*}$, then $h_{2}(x)=x$.

(3) For each $g^{\prime} \in G_{2}^{\prime}$ contained in $\mathscr{U}_{2}^{*}$, there exists a $U \in \mathscr{U}_{2}$ such that $h_{2}\left(g^{\prime}\right) \cup g^{\prime} \subset U$.

Claim. For each $g \in G$ contained in $\mathscr{U}_{1}^{*}$, there exists a $W \in \mathscr{C}_{1}$ such that $g \cup h_{2} h_{1}(g) \subset W$.

Proof of Claim. Suppose $g \in G$ and $g \subset \mathscr{U}_{1}^{*}$. Then there exists $U \in \mathscr{U}_{1}$ such that $h_{1}(g) \cup g \subset U$. If $g \in H_{G_{1}}$ or if $h_{1}(g)$ is not contained in $\mathscr{V}_{2}^{*}$, then $h_{2} h_{1}(g)=h_{1}(g)$, and we are done. Suppose then that $g \notin H_{G_{1}}$ and $h_{1}(g) \cap \mathscr{V}_{2}^{*} \neq \varnothing$. Since $\mathscr{U}_{2}^{\prime}$ is a refinement of $h_{1}\left[\mathscr{U}_{1}\right]$ and $\mathscr{U}_{2}$ is a locally finite star refinement of $\mathscr{L}_{2}^{\prime}$, we may find $U_{2} \in \mathscr{L}_{2}$ and $U_{g}, \in \mathscr{U}_{2}^{\prime}$, where $h_{1}(g)=g^{\prime}$, such that $h_{1}(g) \subset U_{2} \subset \operatorname{St}\left(U_{2}, \mathscr{L}_{2}\right) \subset U_{g^{\prime}}$. We first show that there exists a $V \in \mathscr{U}_{1}$ such that $U_{g}, \subset V$. Of course, $h_{1}(g)=g^{\prime} \subset U_{g^{\prime}}$. Let $V_{1}, V_{2}, \cdots, V_{n}$ be those members of $\mathscr{U}_{1}$ which contain $g$. Then by our construction of $\mathscr{U}_{2}^{\prime}$,

$$
U_{g^{\prime}} \subset h_{1}\left(V_{1}\right) \cap \cdots \cap h_{1}\left(V_{n}\right) \text {. }
$$

Since $h_{1}(g) \subset U_{g^{\prime}}$, it follows that $g \subset V_{1} \cap \cdots \cap V_{n}$. But for at least 
one $i=1,2, \cdots$, or $n, h_{1}(g) \cap g \cup V_{i}$. Therefore, by (5) in our construction of $\mathscr{U}_{2}^{\prime}$, it must be the case that $U_{g^{\prime}}$ is contained in $V_{i}$.

We need only observe now that if $Z \in \mathscr{Q}_{2}$ and $h_{1}(g) \subset Z$, then $Z \subset V_{i}$. This is clear since $Z \subset \operatorname{St}\left(U_{2}, \mathscr{C}_{2}\right) \subset U_{g^{\prime}} \subset V_{i}$. Hence, we have that $\operatorname{St}\left(h_{1}(g), \mathscr{U}_{2}\right)$ is contained in $V_{i}$ and since

$$
h_{2} h_{1}(g) \subset \operatorname{St}\left(h_{1}(g), \mathscr{C}_{2}\right),
$$

the proof of the claim is complete.

We continue inductively. Assume now that covers $\mathscr{C}_{1}^{\prime}, \cdots, \mathscr{C}_{n}^{\prime}$, $\mathscr{U}_{1}, \cdots, \mathscr{C}_{n}, \mathscr{V}_{1}, \cdots, \mathscr{Y}_{n}$ have been defined so that the conditions listed below are satisfied. We denote $h_{k} h_{k-1} \cdots h_{1}$ by $\hat{h}_{k}$, and $h_{0}=\hat{h}_{0}=$ identity. For $i=1,2, \cdots, n$, let $K_{i}=\{p \in M$ : there exists a sequence of nondegenerate elements converging to $p$ where each element is a member of a different $\left.H_{\hat{h}_{i-1}\left[G_{j}\right]}\right\}$.

(1) $\mathscr{C}_{i}^{\prime}=\left\{U_{g^{\prime}}: g^{\prime} \in H_{\hat{h}_{i-1}[G]}\right\}$ is a collection of saturated (with respect to $\left.\hat{h}_{i-1}[G]\right)$ open sets which refines $\hat{h}_{i-1}\left[\mathscr{C}_{i-1}\right]$ and misses $K_{i}$. For each $g^{\prime}, U_{g^{\prime}}$ is chosen to contain $g^{\prime}$ such that

(a) If $\varepsilon_{g^{\prime}}=\min \left\{\operatorname{diam} g^{\prime}, 1 / 2 d\left(g^{\prime}, K_{i}\right) 1 / i\right\}$, then $U_{g^{\prime}} \subset S_{\varepsilon_{g^{\prime}}}\left(g^{\prime}\right)$.

(b) If $g_{j} \in H_{\hat{h}_{i-1}\left[G_{j}\right]}$ and $g_{k} \in H_{\hat{h}_{i-1}\left[G_{k}\right]}(j \neq k)$ and $g_{j}$ and $g_{k}$ are contained in $U_{g^{\prime}}$, then $1 / 2 \operatorname{diam} g_{j}<\operatorname{diam} g_{k}<3 / 2 \operatorname{diam} g_{j}$.

(2) $\mathscr{U}_{i}$ is a saturated open locally finite star refinement of $\mathscr{U}_{i}^{\prime}$ and $\mathscr{V}_{i}=\left\{U \in \mathscr{U}_{i}: U \cap H_{\hat{h}_{i-1}}^{*}\left[\sigma_{i-1}\right] \neq \varnothing\right\}$.

(3) For $i=1,2, \cdots, n$ and $1 \leqq j \leqq i-1$,

$$
h_{j}^{-1} \cdots h_{i-2}^{-1} h_{i-1}^{-1}\left(\mathscr{C}_{i}^{*}\right) \subset S_{1 / i}\left(\hat{h}_{j-1}\left(H_{G}^{*}\right)\right)
$$

and

$$
h_{j}^{-1} \cdots h_{i-2}^{-1} h_{i-1}^{-1}\left(\mathscr{V}_{i}^{*}\right) \subset S_{1 / 2}\left(\hat{h}_{j-1}\left(H_{G_{i}}^{*}\right)\right) \text {. }
$$

(4) For $i=1,2, \cdots, n, h_{i}$ is a closed map from $M$ onto $M$ such that if $G_{i}^{\prime}=\left\{\hat{h}_{i-1}(g): g \in G_{i}\right\}$ then

(1) $G_{i}^{\prime}=\left\{h_{i}^{-1}(x): x \in M\right\}$.

(2) If $x \in M-\mathscr{Y}_{i}^{*}$, then $h_{i}(x)=x$.

(3) For each $g^{\prime} \in G_{i}^{\prime}$ which is contained in $\mathscr{Q}_{i}^{*}$, there exists $U \in \mathscr{U}_{i}$, such that $h_{i}\left(g^{\prime}\right) \cup g^{\prime} \subset U$.

(5) For $i=1,2, \cdots, n$ and $0 \leqq j \leqq i-1$, if $g \in G$ and $\hat{h}_{i-1}(g)$ is contained in $\mathscr{C}_{1}^{*}$, then there exists $U \in \mathscr{Q}_{j+1}$ such that $\hat{h}_{j}(g) \cup$ $\hat{h}_{i}(g) \subset U$.

(6) $\mathscr{U}_{i}^{\prime *} \cap \mathrm{Cl}\left(h_{i-1}\left(H_{G_{1}}^{*} \cup \cdots \cup H_{G_{i-1}}^{*}\right)\right)=\varnothing$.

Step $n+1$. Let $G_{n+1}^{\prime}=\left\{\hat{h}_{n}(g): g \in G_{n+1}\right\}$. A proof similar to that employed in Step 2 shows that $M / G_{n+1}^{\prime}$ is homeomorphic to $M$. Let $K_{n+1}=\{p \in M$ : there exists a sequence of nondegenerate elements converging to $p$ where each element is a member of a different $H_{h_{n}\left[G_{j}\right]}$. 
We construct a saturated (with respect to $\hat{h}_{n}[G]$ ) open refinement of $h_{n}\left[\mathscr{U}_{n}\right]$ which covers $H_{\hat{h}_{n}[G]}$ and misses $K_{n+1}$. Let $g^{\prime}=\hat{h}_{n}(g)$ where $g \in H_{G}-\left(H_{G_{1}} \cup \cdots \cup H_{G_{n}}\right)$. Choose $U_{g^{\prime}}$ to be a saturated open set containing $g^{\prime}$ such that

(1) If $\varepsilon_{g^{\prime}}=\min \left\{\operatorname{diam} g^{\prime}, 1 / 2 d\left(g^{\prime}, K_{n+1}\right), 1 / n+1\right\}$, then $U_{g^{\prime}} \subset S_{\varepsilon_{g^{\prime}}}\left(g^{\prime}\right)$.

(2) If $g_{i} \in H_{\hat{h}_{n}\left[G_{i}\right]}$ and $g_{j} \in H_{\hat{h}_{n}\left[G_{j}\right]}(i \neq j)$ and $g_{i}$ and $g_{j}$ are contained in $U_{g^{\prime}}$, then $1 / 2$ diam $g_{i}<\operatorname{diam} g_{j}<3 / 2 \operatorname{diam} g_{i}$.

(3) For $i=1,2, \cdots, n,\left(h_{i} h_{i+1} \cdots h_{n}\right)^{-1}\left(U_{g^{\prime}}\right) \subset S_{1 / 2 n}\left(\hat{h}_{i-1}(g)\right)$.

(4) For $i=1,2, \cdots, n$, if $U^{i}$ is the intersection of those sets in $\mathscr{U}_{i}$ which contain $\hat{h}_{i-1}(g)$, then

$$
U_{g^{\prime}} \subset \hat{h}_{n}\left(U^{1}\right) \cap h_{n} h_{n-1} \cdots h_{2}\left(U^{2}\right) \cap \cdots \cap h_{n}\left(U^{n}\right) .
$$

(5) For $0 \leqq i<n$, if $\hat{h}_{i}(g) \cup \hat{h}_{n}(g) \subset U \in \mathscr{K}_{n}$, then $U_{g^{\prime}} \subset U$.

(6) $U_{g^{\prime}} \cap \mathrm{Cl} \hat{h}_{n}\left(H_{G_{1}}^{*} \cup \cdots \cup H_{G_{n}}^{*}\right)=\varnothing$.

Let $\mathscr{U}_{n+1}^{\prime}=\left\{U_{g^{\prime}}: g^{\prime} \in H_{G_{n+1}^{\prime}}\right\}$, let $\mathscr{U}_{n+1}$ be a saturated open locally finite star refinement of $\mathscr{U}_{n+1}^{\prime}$, and let $\mathscr{V}_{n+1}=\left\{U \in \mathscr{Q}_{n+1}: U \cap \hat{h}_{n}\left[H_{G_{n+1}}^{*}\right] \neq \varnothing\right\}$. By Lemma 1 there exists a closed map $h_{n+1}$ from $M$ onto $M$ such that

(1) $G_{n+1}^{\prime}=\left\{h_{n+1}^{-1}(x): x \in M\right\}$.

(2) If $x \in M-\mathscr{Y}_{n+1}^{*}$, then $h_{n+1}(x)=x$.

(3) For each $g \in G_{n+1}^{\prime}$ contained in $\mathscr{U}_{n+1}^{*}$, there exists $U \in \mathscr{U}_{n+1}$ such that $g \cup h_{n+1}(g) \subset U$.

Claim. Suppose $g^{\prime}=\hat{h}_{n+1}(g)$ is contained in $\mathscr{K}_{n+1}^{*}(g$ is an element of $G$ ). Suppose $0 \leqq i<n+1$. Then there exists $U \in \mathscr{U}_{i+1}$ such that $g^{\prime} \cup \hat{h}_{i}(g) \subset U$.

A proof patterned after the proof of the Claim in Step 2 may be used to establish this Claim.

Define $h=\operatorname{Lim} \hat{h}_{n}$. To see that $h$ is well defined, we observe that for each $x \in M$, there exists an integer $N$ such that for $n>N$,

$$
\hat{h}_{n}(x)=\hat{h}_{N}(x)=h(x) \text {. }
$$

This is clearly the case if $x \in H_{G}^{*}$, since if $N$ is the first integer such that $x \in H_{G_{N}}^{*}$, then $h_{N}(x)$ does not belong to the succeeding $\mathscr{U}_{n}^{*}$, and, hence, is left fixed. If $x \notin \mathrm{Cl} H_{G}^{*}$ then choose $N$ such that

$$
d\left(x, \mathrm{Cl} H_{G}^{*}\right)>\frac{1}{N} .
$$

Then $h_{N}(x) \notin \mathscr{Q}_{N+1}^{*}$ (see (3) in the inductive Step $n+1$ ) and it follows that $h(x)=\hat{h}_{n}(x)$ for each $n>N$. Finally, consider the case where $x \in\left(\mathrm{Cl} H_{G}^{*}\right)-H_{G}^{*}$. If there exists an open set $U$ such that $U \cap H_{G_{i}}^{*}=$ $\varnothing$ for all but a finite number of $i$, then it again follows from (3) of Step $n+1$ that the required positive integer $N$ exists. On the other 
hand, if no such $U$ exists, then there is a sequence $\left\{g_{n_{i}}\right\}$ of nondegenerate elements from distinct decompositions $G_{i_{n}}$ which converges to $x$. But it was noted in Step 1 that in this case $x \notin \mathscr{Q}_{1}^{*}$ and thus $h(x)=x$.

We next show that $h$ is continuous. Suppose $\left\{x_{i}\right\}$ is a sequence of points in $M$ converging to a point $x$. If there exists an open set $U$ containing $x$ such that $U \cap H_{G_{i}}^{*}=\varnothing$ for all but at most a finite number of $i$, then it follows again from (3) of the induction Step $n+1$ that $\left\{h\left(x_{i}\right)\right\}$ converges to $h(x)$. If no such $U$ exists, then there are two cases to consider.

Case 1. $x \in\left(\mathrm{Cl} H_{G}^{*}-H_{G}^{*}\right)$. Suppose for each $i, x_{i} \in g_{n_{i}} \in G_{n_{i}}$. We may assume that the $x_{i}$ lie in $\mathscr{U}_{1}^{*}$ since if not $h\left(x_{i}\right)=x_{i}$. But as it was observed in Step 1, since the sequence $\left\{g_{n_{i}}\right\}$ converges to $x$, we have that the corresponding sequence $\left\{\operatorname{St}\left(g_{n_{i}}, \mathscr{U}_{1}\right)\right\}$ also converges to $x$. It follows from the Claim in Step $n+1$, that $h\left(x_{i}\right) \in \operatorname{St}\left(g_{n_{i}}, \mathscr{U}_{1}\right)$, and, therefore, $\left\{h\left(x_{i}\right)\right\}$ converges to $h(x)$.

Case 2. $x \in H_{G}^{*}$. Let $n$ be the first integer such that $x \in g_{n} \in H_{G_{n}}$. But then $\hat{h}_{n}\left(g_{n}\right)$ is a point and our construction in the inductive steps reduces this case to Case 1 .

That $h$ is onto may be seen by the following argument. Suppose $p$ is a point in $M$. We assume that $p \in g^{\prime} \in G$ where $g^{\prime} \subset \mathscr{U}_{1}^{*}$ (if not, $h(p)=p$ ). For each positive integer $i$, there exists a point $x_{i}$ in $\mathscr{U}_{1}{ }^{*}$ such that $h_{i}\left(x_{i}\right)=p$. It follows from the Claim in Step $n+1$ that for each $i, x_{i} \in \operatorname{St}\left(g^{\prime}, \mathscr{U}_{1}\right)$. Since St $\left(g^{\prime}, \mathscr{C}_{1}\right)$ has compact closure (see Step 1), there exists an accumulation point $x$ of the sequence $\left\{x_{i}\right\}$. For simplicity of notation let us assume that $\left\{x_{i}\right\}$ converges to $x$. We show that $h(x)=p$.

Let $g \in G$ be the member of the decomposition which contains $x$. Choose $N$ large enough so that $\hat{h}_{n}(g)=h(g)$ for each $n \geqq N$. First we suppose that there exists a positive integer $K \geqq N$ such that for $n \geqq K, S_{1 / K}(g) \cap H_{G_{n}}^{*}=\varnothing$. Of course, the sequence $\left\{\hat{h}_{K}\left(x_{i}\right)\right\}$ converges to $\hat{h}_{K}(x)$. But it follows from (3) of Step $n+1$, that for $i$ sufficiently large, we will have $\hat{h}_{k}\left(x_{i}\right)=\hat{h}_{i}\left(x_{i}\right)=h\left(x_{i}\right)$. Thus $h(x)=p$, since $\hat{h}_{i}\left(x_{i}\right)=p$ for all $i$.

Now suppose that each open set containing $x$ intersects an infinite number of the $H_{G_{i}}^{*}$, and, hence, each open set containing $\hat{h}_{N}(x)$ will also intersect infinitely many of the sets $H_{\hat{h}_{N}\left[\sigma_{i}\right]}^{*}$. Thus, $\hat{h}_{N}(x)$ belongs to $K_{n+1}$ (see Step $n+1$ ). Since $\left\{\hat{h}_{N}\left(x_{i}\right)\right\}$ converges to $\hat{h}_{N}(x)$, it follows from conditions (1) and (3) of Step $n+1$ that the sequence

$$
\left\{\operatorname{St}\left(\hat{h}_{N}\left(x_{i}\right), \mathscr{U}_{N}\right)\right\}
$$

also converges to $\hat{h}_{N}(x)$.

But the Claim in this step ensures that for $j>N, \hat{h}_{j}\left(x_{i}\right) \cup \hat{h}_{N}\left(x_{i}\right)$ belongs to $\mathrm{St}\left(\hat{h}_{N}\left(x_{i}\right), \mathscr{C}_{N}\right)$. In particular then for $i>N$, 


$$
\hat{h}_{i}\left(x_{i}\right) \cup \hat{h}_{N}\left(x_{i}\right) \subset \operatorname{St}\left(\hat{h}_{N}\left(x_{i}\right), \mathscr{C}_{N}\right),
$$

and since, $\hat{h}_{i}\left(x_{i}\right)=p$, it again follows that $h(x)=p$. Thus $h$ is an onto map.

It is easily seen from our construction of $h$ that $G=\left\{h^{-1}(x): x \in M\right\}$.

Finally, we must show that $h$ is closed. It suffices to show that if $K$ is a compact subset of $M$, then $h^{-1}(K)$ is also compact. Since $h$ is onto, for each $x \in K$, there exists a unique element $g_{x} \in G$ such that $h\left(g_{x}\right)=x$. If $g_{x} \subset \mathscr{Q}_{1}^{*}$, let $U_{g_{x}}$ be a member of $\mathscr{C}_{1}$ which contains $g_{x}$. If $g_{x}$ is not contained in $\mathscr{U}_{1}^{*}$ let $U_{g_{x}}$ be an open set containing $g_{x}$ with compact closure. Note that it follows from Step 1 that if $g_{x}$ is contained in $\mathscr{U}_{1}^{*}$, then $\operatorname{St}\left(U_{g_{x}}, \mathscr{U}_{1}\right)$ has compact closure. Since if $g_{x} \subset \mathscr{U}_{1}^{*}$, then $g_{x} \cup h\left(g_{x}\right) \subset \operatorname{St}\left(U_{g_{x}}, \mathscr{U}_{1}\right)$, and if $g_{x}$ is not contained in $\mathscr{Q}_{1}^{*}$, then $h\left(g_{x}\right)=g_{x}$, the collection $\left\{U_{g_{x}}: x \in K\right\}$ is an open cover of $K$. Let $U_{g_{x_{1}}}, \cdots, U_{g_{x_{n}}}$, be a finite subcover of $K$, where the first $i$ terms are members of $\mathscr{U}_{1}$. To finish the proof we need only observe that

$$
h^{-1}(K) \subset \operatorname{St}\left(g_{x_{1}}, \mathscr{C}_{1}\right) \cup \cdots \cup \operatorname{St}\left(g_{x_{i}}, \mathscr{C}_{1}\right) \cup U_{g_{x_{i+1}}} \cup \cdots \cup U_{g_{x_{n}}}
$$

and that the right hand set has compact closure. Thus, the conditions of property $S$ have been satisfied, and, hence, $M / G$ is homeomorphic to $M$.

A decomposition of a metric space is said to be nondegenerately continuous if for each $g \in G, H_{G} \cup\{g\}$ is continuous at $g$.

COROLlary 1. Suppose $G$ is a cellular nondegenerately continuous upper semicontinuous decomposition of $E^{3}$. Suppose there exists a countable number of planes in $E^{3}, Q_{1}, Q_{2}, \ldots$ such that for each $g \in H_{G}, g$ is contained in at least one of these planes. Then $E^{3} / G$ is homeomorphic to $E^{3}$.

Proof. For $i=1,2, \cdots$, let $G_{i}$ be the decomposition of $E^{3}$ such that $H_{G_{i}}=\left\{g \in H_{G}: g \subset Q_{i}\right\}$. Then $E^{3} / G_{i}$ is homeomorphic to $E^{3}$ [3], and since it is readily verified that $G_{1}, G_{2}, \cdots$ satisfy the conditions of Theorem $1, E^{3} / G$ is homeomorphic to $E^{3}$.

\section{REFERENCES}

1. S. Armentrout, Concerning cellular decompositions of 3-manifolds with boundary, Trans. Amer. Math. Soc., 137 (1969), 231-236.

2. R. H. Bing, Point-like decompositions of $E^{3}$, Fund. Math., 45 (1962), 431-453.

3. E. Dyer and M. E. Hamstrom, Completely regular mappings, Fund. Math., 45 (1958), 103-118.

4. J. Lamoreaux, Decompositions of metric spaces with a 0-dimensional set of nondegenerate elements, Notices Amer. Math. Soc., 14 (1967), 89. 
5. L. Siebenmann, Approximating cellular maps by homeomorphisms, Notices Amer. Math. Soc., 17 (1970), 532.

6. W. Voxman, Nondegenerately continuous decompositions of 3-manifolds, Fund. Math., 68 (1970), 307-320.

7. On the union of certain cellular decompositions of 3-manifolds, Illinois J. Math., 15, No. 3, (1971), 387-392.

Received March 6, 1972.

UNIVERSITY OF IDAHO 



\section{PACIFIC JOURNAL OF MATHEMATICS}

\section{EDITORS}

D. Gilbarg and J. Milgram

Stanford University

Stanford, California 94305

R. A. Beaumont

University of Washington

Seattle, Washington 98105
J. DUGUNDJI* Department of Mathematics

University of Southern California Los Angeles, California 90007

RICHARD ARENS

University of California

Los Angeles, California 90024

\section{ASSOCIATE EDITORS}

E. F. BeCKenbach

B. H. NeUMaNN

F. WOLF

K. Yoshida

\section{SUPPORTING INSTITUTIONS}

UNIVERSITY OF BRITISH COLUMBIA

UNIVERSITY OF SOUTHERN CALIFORNIA

CALIFORNIA INSTITUTE OF TECHNOLOGY

UNIVERSITY OF CALIFORNIA

MONTANA STATE UNIVERSITY

STANFORD UNIVERSITY

UNIVERSITY OF TOKYO

UNIVERSITY OF NEVADA

UNIVERSITY OF UTAH

NEW MEXICO STATE UNIVERSITY

WASHINGTON STATE UNIVERSITY

OREGON STATE UNIVERSITY

UNIVERSITY OF OREGON

OSAKA UNIVERSITY

UNIVERSITY OF WASHINGTON

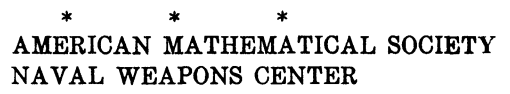

* C. DePrima will replace J. Dugundji until August 1974. 


\section{Pacific Journal of Mathematics}

\section{Vol. 47, No. $1 \quad$ January, 1973}

K. Adachi, Masuo Suzuki and M. Yoshida, Continuation of holomorphic

mappings, with values in a complex Lie group ....................

Michael Aschbacher, A characterization of the unitary and symplectic groups

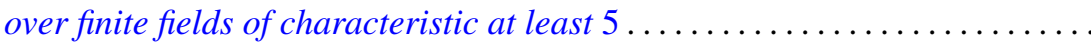

Larry Eugene Bobisud and James Calvert, Energy bounds and virial theorems for abstract wave equations....................................

Christer Borell, A note on an inequality for rearrangements ................

Peter Southcott Bullen and S. N. Mukhopadhyay, Peano derivatives and general

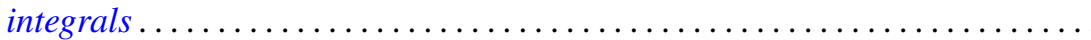

Wendell Dan Curtis, Yu-Lee Lee and Forrest Miller, A class of infinite dimensional subgroups of $\operatorname{Diff}^{r}(X)$ which are Banach Lie groups .........

Paul C. Eklof, The structure of ultraproducts of abelian groups ...............

William Alan Feldman, Axioms of countability and the algebra $C(X) \ldots \ldots \ldots$

Jack Tilden Goodykoontz, Jr., Aposyndetic properties of hyperspaces...........

George Grätzer and J. Płonka, On the number of polynomials of an idempotent algebra. II ...........................................

Alan Trinler Huckleberry, The weak envelope of holomorphy for algebras of

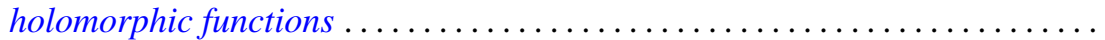

John Joseph Hutchinson and Julius Martin Zelmanowitz, Subdirect sum decompositions of endomorphism rings . . . . . . . . . . . . . . . .

Gary Douglas Jones, An asymptotic property of solutions of

$y^{\prime \prime \prime}+p y^{\prime}+q y=0$.

Howard E. Lacey, On the classification of Lindenstrauss spaces .

Charles Dwight Lahr, Approximate identities for convolution measure algebras.

George William Luna, Subdifferentials of convex functions on Banach

spaces.

Nelson Groh Markley, Locally circular minimal sets. .

Robert Wilmer Miller, Endomorphism rings of finitely generated projective modules

Donald Steven Passman, On the semisimplicity of group rings of linear

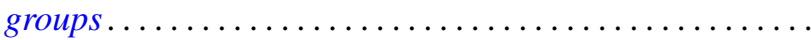

Bennie Jake Pearson, Dendritic compactifications of certain dendritic spaces.

Ryōtarō Satō, Abel-ergodic theorems for subsequences ...... .

Henry S. Sharp, Jr., Locally complete graphs. . .

Harris Samuel Shultz, A very weak topology for the Mikusinski field of

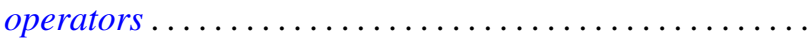

Elena Stroescu, Isometric dilations of contractions on Banach spaces ...

Charles W. Trigg, Versum sequences in the binary system ... . .

William L. Voxman, On the countable union of cellular decompositions of n-manifolds 